\title{
Uboreshaji wa shughuli za uchimbaji mdogo wa madini nchini Tanzania kupitia ubia na wawekezaji
}

\section{Fursa na changamoto}

George Schoneveld (CIFOR), Maisory Chacha (COWI), Maria Njau (COWI), Xiaoxue Weng (IIED), Jesper Jonsson (COWI)

\section{Ujumbe muhimu}

- Wawekezaji wa kigeni wanazidi kuingia ubia na wachimbaji wadogo ili kupata miliki na hifadhi za madini zilizopo kwenye mazingira hatarishi na yenye gharama kubwa.

- Hii imechangia ukuaji wa shughuli za wachimbaji wadogo na kusambaza teknolojia katika maeneo yao ya uchimbaji. Hata hivyo ukuaji huu unaweza kuvuruga mpangilio wa ugawanaji faida uliopo kati ya wachimbaji wadogo na wamiliki wa mashimo/leseni.

- Ukuaji wa uchimbaji mdogo unaotokana na ongezeko la mtaji na usambaaji wa teknolojia umeambatana na hatarishi mbalimbali za kimazingira na usalama na afya kazini.

- Uwezo mdogo wa taasisi za serikali za ngazi ya chini na ukosefu wa uratibu baina ya taasisi umechangia kudhorotesha juhudi za serikali katika kufuatilia na kuboresha usalama na afya kazini na mazingira katika migodi iliyo chini ya ubia.

- Kuna umuhimu wa kuwepo kwa majadiliano ya kisera ya haraka juu ya faida na athari za ubia na jinsi ya kuwezesha ubia huu ili kuboresha sekta ya madini na kuleta maendeleo endelevu katika jamii za uchimbaji vijijini.

- Uratibu wenye ufanisi katika taasisi muhimu za serikali unahitajika kwa haraka, hasa kwenye ngazi ya chini, ili kukabiliana na athari katika mazingira na usalama wa Wafanyakazi zinazotokana na migodi midogo yenye shughuli za kisasa za uchimbaji.

\section{Utangulizi}

Uchimbaji mdogo wa vito vya thamani kwa muda mrefu umekuwa ni nguzo muhimu ya uchumi wa maeneo ya vijijini nchini Tanzania (Chachage 1995; Fischer et al. 2009). Ingawa mchango wa sekta ya uchimbaji mdogo kwenye kuzalisha madini na kuliingizia taifa fedha za kigeni ni mdogo kulinganisha na uchimbaji mkubwa, inakadiriwa kwamba uchimbaji mdogo unatoa ajira za moja kwa moja kwa Watanzania wanaokadiriwa kufika 680,000 ukilinganisha na Watanzania takribani 8,800 walioajiriwa na wachimbaji wakubwa (URT 2011). Inakadiriwa pia kila mchimbaji mdogo mmoja huzalisha ajira zisizo za moja kwa moja nne, hivyo kuifanya sekta ya uchimbaji mdogo kuajiri Watanzania wapatao milioni tatu na ni njia ya kujipatia kipato kikubwa kwa wakazi wengi wa vijijini (Jønsson na Fold 2011).
Ingawa uchimbaji mdogo una mchango mkubwa kwenye maisha ya wakazi wengi vijijini, sekta hii imekuwa ikilalamikiwa kuleta athari katika mazingira na jamii. Hii inachangiwa na kushindwa kufuata viwango vya afya na usalama (Hinton 2005), kuchangia uharibifu wa mazingira (Kitula 2006), chanzo cha ajira kwa watoto (HRW 2013), kuleta matatizo katika jamii yanayotokana na mabadiliko ya demografia, ukosefu wa kipato na ajira (Bryceson na Jønsson 2010), na migogoro ya ardhi (Lange 2011; Carstens na Hilson 2009). Ingawa Sheria na Kanuni zinazoongoza sekta ya uchimbaji madini nchini zimetungwa vizuri, kiuhalisia, shughuli nyingi za wachimbaji wadogo sio rasmi na hufanyika maeneo ambayo hayawezi kufikika kiurahisi na mamlaka za serikali. Kwa kuwa mamlaka nyingi za udhibiti hazina rasilimali wala motisha ya kusimamia utekelezaji wa kanuni zilizopo kwa ufanisi, hupelekea sekta ya uchimbaji mdogo kutoadhibiwa kwa kushindwa kufuata kanuni zilizowekwa. Zaidi ya hayo, kwa kuwa upatikanaji wa 
fedha na vifaa vya kisasa vya uzalishaji ni mdogo na uhusiano na wachimbaji wakubwa pia kuwa mdogo, wachimbaji wadogo walio wengi hutegemea vifaa vya kizamani na kujihusisha na shughuli za uzalishaji zisizokuwa endelevu. Matokeo yake wachimbaji hushindwa kuendeleza miundombinu muhimu ya uchimbaji na hukabiliwa na ufanisi mdogo kiuchumi na kupata kiwango kidogo cha madini kwenye kuchenjua. Kwa sababu hizi, sekta ya uchimbaji mdogo bado haijaweza kufikia uwezo wake kamili.

Tofauti na nchi kama Ghana na Zimbabwe, Tanzania inakosa sekta ya uchimbaji ya ngazi ya kati inayosaidia kuunganisha uchimbaji mdogo na uchimbaji mkubwa. Kwa miaka ya hivi karibuni, kumekuwa na ongezeko kubwa la kampuni za kigeni zinazojishughulisha moja kwa moja na uchimbaji mdogo. Wawekezaji wa kigeni wamehamasika kuona fursa ya kushirikiana na wachimbaji wadogo badala ya kuanzisha shughuli zao wenyewe. Hii inatokana na gharama kubwa na changamoto nyingine za kuanzisha shughuli za uchimbaji na kupungua kwa upatikanaji wa miliki za uchimbaji katika maeneo yenye hifadhi kubwa ya madini. Wawekezaji hao huingiza mtaji na teknolojia ambayo ina uwezo mkubwa wa kutatua baadhi ya changamoto za utendaji katika sekta ya uchimbaji mdogo.

Waraka huu unawasilisha matokeo ya utafiti uliochunguza kwa kina:

1. mienendo ya ubia kati ya wachimbaji wadogo na wawekezaji wa kigeni

2. mchango wa ubia huu katika kuchochea maendeleo ya jamii na kuboresha uchimbaji mdogo, vile vile, athari zake katika mazingira, na

3. ubia huu unadhibitiwa kivipi na kwa kiwango gani na serikali ya Tanzania.

Waraka huu unahitimisha kwa kutoa mapendekezo mbalimbali ya udhibiti ili serikali zinazokumbana na matatizo ya aina hii kama zinaweza kuyazingatia katika juhudi zao za kurasimisha na kudhibiti athari za ubia kati ya wawekezaji wa kigeni na wazalishaji wasio rasmi.

\section{Mbinu}

Utafiti huu ulifanyika katika wilaya nne zilizochaguliwa baada ya ziara ya awali katika Mikoa ya Mwanza, Geita, Arusha, Singida, Dodoma, Kilimanjaro, Kigoma, Mbeya, na Katavi, ambapo watoa taarifa muhimu 123 kutoka sekta binafsi na serikalini walihojiwa. Wilaya zilizochaguliwa ni Geita (Mkoa wa Geita), Chunya (Mkoa wa Mbeya), Mwanga (Mkoa wa Kilimanjaro), na Mpwapwa (Mkoa wa Dodoma). Utafiti katika wilaya za Geita na Chunya ulilenga shughuli za uchimbaji mdogo wa dhahabu, ambapo sampuli tano za ubia kati ya wachimbaji wadogo na wawekezaji wa kigeni zilichambuliwa. Kwa upande mwingine, utafiti katika wilaya za Mwanga na Mpwapwa ulilenga maeneo ya uchimbaji wa shaba, ambapo pia sampuli tano za ubia zilichambuliwa. Utafiti ulichagua kwa makusudi maeneo yenye sekta mbili tofauti za dhahabu na shaba ili kuonesha utofauti uliopo baina ya sekta hizo mbili.

Katika kila eneo, mahojiano na watoa taarifa muhimu (89 kwa ujumla) yalifanyika na wawekezaji, wilayani, serikali za mtaa, viongozi wa vijiji, na wadau wengine kwenye mnyororo wa thamani (mfano, wakala/wauzaji, wasambazaji wa vifaa, na wachenjuaji). Lengo lilikuwa kutambua mchango wa shughuli za uchimbaji kwenye kuleta maendeleo endelevu katika mnyororo wa thamani katika maeneo husika na mienendo ya kisiasa na kiuchumi. Vilevile, katika sampuli nne za ubia kati ya sampuli kumi, jumla ya madodoso 155 yalijazwa ambapo wachimbaji walishiriki moja kwa moja. Pia majadiliano katika makundi mjadala 19 na wadau wengine katika jamii kama vijana na wanawake yalifanyika. Matokeo ya tafiti katika wilaya hizi nne yalitumika kusanifu uchambuzi wa kisiasa na kiuchumi uliofutia. Hii ilijumuisha mahojiano na taasisi tisa katika ngazi ya serikali kuu jijini Dar es Salaam na taasisi 13 katika mikoa na wilaya.

\section{Maelezo ya jumla ya ubia}

Sekta ya uchimbaji mdogo wa dhahabu ilianza kupata kiwango kikubwa cha uwekezaji kutoka nje ya nchi katikati hadi mwishoni mwa miaka ya 2000 ambapo wawekezaji wa kigeni walianzisha mitambo ya kuchenjua magwangala, hasahasa katika wilaya ya Geita. Ingawa mitambo hiyo ilitengeneza nafasi kwa wachimbaji wadogo kunufaika na magwangala ambayo kwa muda mrefu yaliachwa kama uchafu, uwekezaji huu haukuhusisha ubia na wachimbaji wadogo wala utaratibu wa uhamishaji wa teknolojia. Baada ya bei ya magwangala kuongezeka kutokana na kuongezeka kwa ushindani na kupungua kwa uzalishaji, wawekezaji wengi wenye mitambo ya kuchenjua walianza kuangalia fursa za kuwa na uwezo mkubwa wa kumiliki dhahabu kwa kujihusisha na uchimbaji wenyewe.

Kwenye sekta ya uchimbaji mdogo wa shaba, ambayo haijakua nchini, wawekezaji wa kigeni waliingia kama wawakilishi wa wanunuzi. Kupanda kwa bei ya shaba katika soko la dunia mwanzoni mwa miaka ya 2010 kulipelekea ongezeko kubwa la ununuzi na uuzaji wa madini ya shaba na makinikia yake nje ya nchi kutoka kwa wachimbaji wadogo, huku wanunuzi wakubwa wakiwa Wachina wenye kufahamu masoko nchini kwao. Hata hivyo, kuanzia mwaka 2014 wanunuzi wengi walianza kujiondoa kufuatia kushuka kwa bei na ubora wa shaba. Wanunuzi ambao hawakujiondoa, walianza kuangalia fursa za kujihusisha na uchimbaji moja kwa moja, kama ilivyokuwa kwa wawekezaji kwenye sekta ya dhahabu.

Katika sekta zote za dhahabu na shaba, wawekezaji wa kigeni waliona uhitaji wa haraka wa kujishughulisha moja kwa moja na uchimbaji hivyo kuamua kuingia ubia na wamiliki mbalimbali wa leseni ya uchimbaji mdogo (PML) ambayo hutolewa kwa raia wa Tanzania pekee. Ingawa Sheria ya madini inakataza raia wa kigeni kujihusisha na shughuli za uchimbaji kwa kutumia leseni ya uchimbaji mdogo, wawekezaji walio wengi wa kigeni katika ubia wao na wazawa hufanya shughuli zote za uchimbaji na uchenjuaji, wakitumia utaratibu na mashine za kisasa. Kwa wawekezaji wengi, mbinu hii ya kutumia leseni ya uchimbaji mdogo huwapunguzia gharama kubwa, muda mwingi wa ufuatiliaji na urasimu utakaojitokeza kwa kufuata utaratibu rasmi wa ukataji wa leseni ya uchimbaji mkubwa (ML). Hii ni kwa sababu, kuanzisha mgodi kwa kutumia leseni ya uchimbaji mkubwa huwalazimu wao kuwekeza zaidi kwenye kufanya utafutaji wa madini, upembuzi yakinifu, na kufanya Tathmini ya Athari kwa Mazingira (TAM) ambavyo kwa pamoja huchukua zaidi ya miaka mitano kabla ya uwekezaji katika uchimbaji haujaanza. Vilevile, katika uchimbaji wa dhahabu, ni maeneo machache ambayo hayana leseni za utafutaji madini na leseni za uchimbaji. Hii ni kwa sababu wachimbaji wakubwa watano pekee wa dhahabu wanashikilia maeneo mengi yenye kuthibitika kuwa na hifadhi kubwa ya dhahabu huku maeneo mengine yaliyobaki yakishikiliwa na wamiliki wa leseni za uchimbaji mdogo, na wamiliki wa leseni za utafutaji madini ambazo humilikiwa na Watanzania waishio mijini na raia wa kigeni. 
Hivyo basi, kupata maeneo yenye kuthibitika kuwa na hifadh kubwa ya dhahabu kunahitaji kushirikisha wamiliki wa leseni waliopo.

\section{Mchanganyiko wa athari kwa ajira, mazingira, na maendeleo ya sekta}

Sampuli tisa kati ya 10 za ubia kati ya wachimbaji wadogo na wawekezaji wa kigeni zilizochambuliwa zimeonesha kwamba hakuna mwekezaji aliyeamua kubakiza au kuendeleza muundo wa uchimbaji mdogo uliokuwepo. Kwa kawaida, muundo wa uchimbaji mdogo hujumuisha ngazi tatu za majukumu na ugawanyaji wa faida kati ya mmiliki wa leseni ya uchimbaji mdogo (mwenye majukumu ya kisheria na usalama kwa ujumla), mmiliki wa shimo (hutoa ufadhili na kuendeleza shimo) na wachimbaji (wana majukumu ya kuchimba na kuchenjua) (tizama Kielelezo 1 chenye kuonesha muundo huu na soma Bryceson et al. (2014) na
Kulindwa et al. (2003) kwa maelezo zaidi). Kwa kupitia utaratibu huu, wachimbaji wadogo hawalipwi mishahara ila huwa wanahisa wenye kuweza kupata faida kubwa kwenye mgodi unaozalisha sana na hubeba hasara ikiwa mgodi hautazalisha. Kwa upande mwingine, kwenye migodi inayofanya kazi kwa mfumo wa ubia, utaratibu wa ufanyaji kazi ni rasmi zaidi ambapo wachimbaji ni wafanyakazi wanaopokea mishahara (Kielelezo 2). Ingawa utaratibu uliopo kwenye ubia unawahakikishia wachimbaji kipato kupitia mshahara huku baadhi wakipata marupurupu, wachimbaji hawatilii mkazo utendaji wa mgodi kwani hawana fursa ya kujipatia fedha nyingi kutokana na ongezeko la uzalishaji. Ingawa jamii hufaidika na uwekezaji kwenye miundombinu ya uchimbaji lakini zaidi ya ajira, jamii zilizo chini ya ubia huu hupata faida chache za moja kwa moja kutokana na utajiri wa madini uliopo katika ardhi yao.

Kwa kuwa uchimbaji mkubwa unategemea sana mtaji wa kununua vifaa vya kisasa kuliko wingi wa watu, kukua kwa ubia kati ya wachimbaji wadogo na wawekezaji wa kigeni kumewanufaisha zaidi wawekezaji wenye mtaji mkubwa kwa faida kwenda kwao

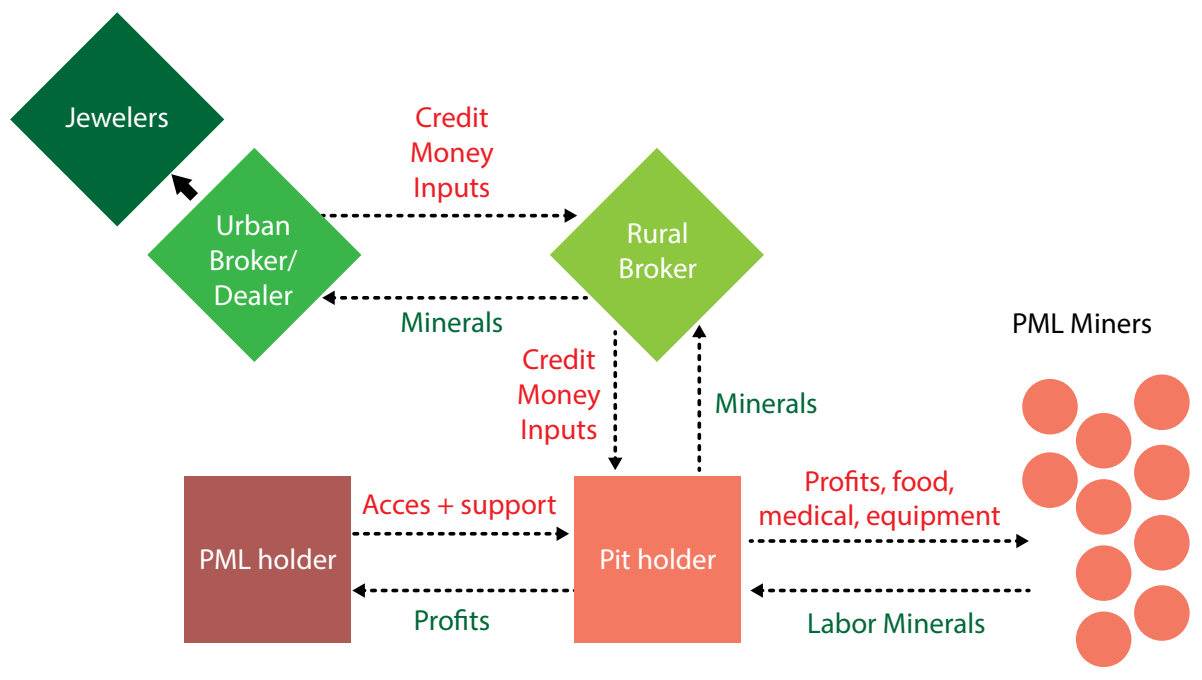

Kielelezo 1. Modeli ya uendeshaji shughuli za uchimbaji mdogo

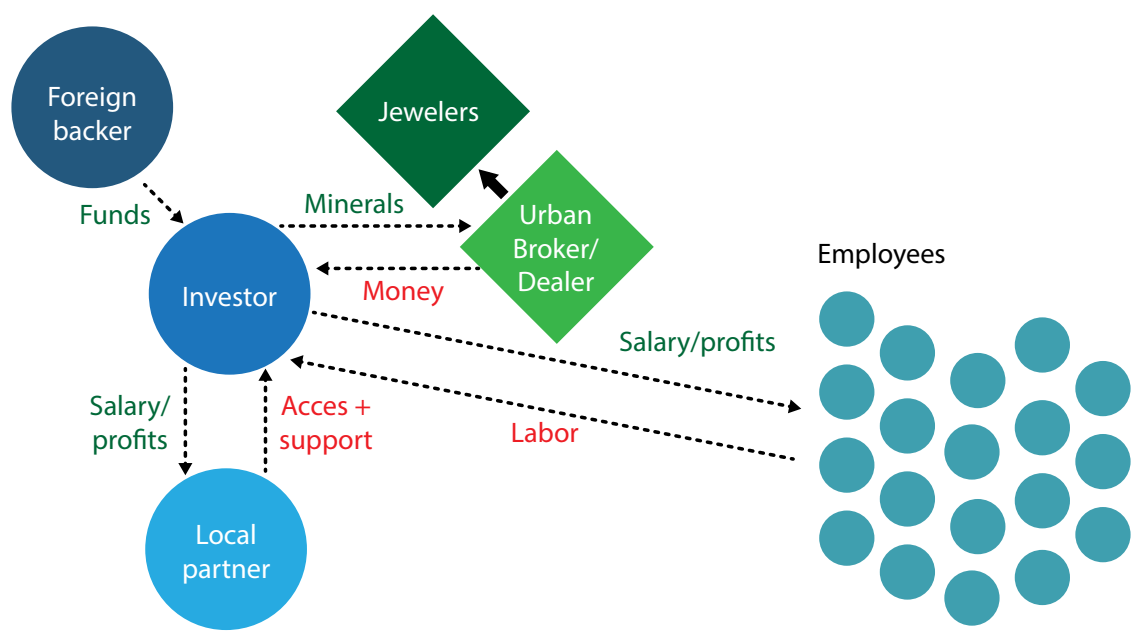


huku kukitishia kupunguza nafasi za ajira kwa wakazi wa vijijini kwa miaka ya mbeleni. Kwa upande mwingine, utaratibu huu wa ubia una fursa ya kuleta matokeo chanya kwa kuendeleza leseni ambazo hazitumiki na kuzalisha ajira nje ya sekta ya kilimo vijijini. Hii ni kwa sababu kati ya zaidi ya leseni 30,000 za uchimbaji mdogo, ni asilimia chini ya 10 pekee ndio zinaendelezwa. Hata hivyo, mafanikio ya ubia huu umeonesha utofauti kulingana na maeneo. Kwa mfano, wakati migodi ya dhahabu iliyo chini ya ubia kwenye wilaya za Geita na Chunya inafanya kazi, migodi mingi kwenye maeneo yenye shaba (wilaya za Mwanga na Mpwapwa) imekuwa haifanyi kazi.

Uendelezaji wa ujuzi kwa wafanyakazi ni mdogo sana katika migodi mingi kwani kazi nyingi hazihitaji ujuzi, na zile zenye ujuzi hufanywa na wafanyakazi wa kigeni. Ni mgodi mmoja tu ambao una programu ya mafunzo, hata hivyo, uongozi wa mgodi unadai pindi wafanyakazi wanapomaliza mafunzo yao huacha kazi na kuchukua kazi kwingine kwenye malipo mazuri. Hii inadhihirisha ukosefu wa misingi ya kiuchumi kwa wawekezaji kuendelea na programu za mafunzo kwa wafanyakazi wake. Vilevile, hakuna mgodi wowote uliotengeneza ushirikiano wa karibu na migodi mingine midogo ili kutoa fursa ya kuhamisha teknolojia au mtaji. Matokeo yake ubia haujaweza kuleta mabadiliko chanya kwa kusambaza teknolojia zaidi ya maonesho ya teknolojia ya kisasa. Hatahivyo, wawekezaji wameweza kutoa michango muhimu kisekta kupitia teknolojia, kuwekeza mtaji na kuongeza thamani kupitia matumizi ya vifaa vya kisasa vya uchenjuaji. Zaidi ya hayo, wachimbaji wadogo walioboresha shughuli zao za uchimbaji walibainisha kwamba uwepo wa migodi iliyo chini ya ubia ni kielelezo muhimu cha kuonesha faida ya aina hii mpya ya ufanyaji biashara. Ingawa uharibifu wa mazingira, afya na usalama kazini na changamoto nyingine za kufuata sheria na kanuni zilizoko ni baadhi ya changamoto zinazokabili ubia, kwa kutumia njia za kisasa za uchimbaji na uchenjuaji, wawekezaji kwenye ubia huweza kufanya vizuri kuliko wachimbaji wadogo. Hii inahusisha, kwa mfano, matumizi ya teknolojia ya kiwango cha juu inayopunguza hatarishi za uchafuzi unaotokana na matumizi ya sianidi na zebaki; na matumizi ya mbinu za uchimbaji zenye ufanisi ambazo hupunguza utegemezi wa Uchimbaji wa Mgodi wa Wazi ambao una uwezo mkubwa wa kuhusisha ukataji miti, upotevu wa rutuba ya udongo, na uchafuzi wa hewa kuliko uchimbaji wa chini ya ardhi. Vilevile, tofauti na wachimbaji wadogo, wawekezaji wengi wamechangia katika shughuli za maendeleo ya jamii katika maeneo yao

\section{Mienendo ya udhibiti na changamoto}

Uchambuzi wa jinsi ubia unavyodhibitiwa na kwa kiwango gani umeonesha changamoto mbalimbali (hasahasa za kitaasisi). Changamoto hizi zinahitaji kupewa umakini mkubwa kama sekta ya umma inaweza kuchangia kwa kiasi kikubwa kuboresha ubia ili kuongeza ufanisi wa utendaji katika sekta ya uchimbaji mdogo kwenye nyanja za mazingira, jamii na uchumi.

Wilaya, serikali za mitaa, wizara na taasisi zake huchukulia kwamba kanuni zinazoongoza umiliki wa leseni ya uchimbaji mdogo ni kali sana na zisizoweza kwenda sambamba na vipaumbele vya maendeleo kwenye jamii. Leseni nyingi za uchimbaji mdogo zinahodhiwa bila maendelezo au zinashindwa kuzalisha kutokana na uwezo na rasilimali ndogo za kuwezesha uchimbaji.
Wadau wengi wa serikali huchukulia uboreshaji wa shughuli za uchimbaji, kwa kutumia leseni ya uchimbaji mdogo, unaofanywa na wawekezaji kama hatua nzuri ya kuendeleza sekta ya uchimbaji mdogo na uchumi wa jamii zinazowazunguka kwa ujumla. Kwa misingi hiyo, wawekezaji huachwa waendelee na shughuli zao huku kukiwa hakuna ushahidi wowote unaoonesha serikali kuwabana kufuata kanuni za uchimbaji (zinazokataza raia wa kigeni kufanyia kazi leseni ya uchimbaji mdogo) au kukata leseni ya uchimbaji mkubwa (ML) itakayofanya shughuli zao ziende kisheria. Uzito huu wa ufuatiliaji hutokana na hofu kwamba kwa kuwalazimisha wawekezaji kukata leseni ya uchimbaji mkubwa kutawakatisha tamaa kutokana na gharama za fedha na muda zinazohitajika kuanzisha mgodi kwa kutumia leseni hiyo.

Uwepo wa maslahi binafsi pia huchagia katika haya yote. Kwa mfano, baadhi ya maafisa katika wilaya na ofisi za madini ambao hufahamu shughuli za uchimbaji kwenye maeneo yao wamekuwa wakiwasaidia wawekezaji, kwa njia isiyo rasmi, kutambua wamiliki wa leseni za uchimbaji mdogo na kufanya nao mazungumzo. Vilevile, wawekezaji wengi walikosea mwanzoni kwa kudhani kwamba kwa kufanyia kazi leseni ya uchimbaji mdogo kutawafanya wasidhibitiwe na mamlaka husika kwenye baadhi ya mambo. Kwa mfano, kuajiri vibarua isivyo rasmi, kutokata leseni ya uchimbaji mkubwa, na kutotilia mkazo kanuni za usimamizi wa mazingira, kodi, uhamiaji na usalama na afya sehemu za kazi. Wawekezaji wengi wamedai kwamba jambo hili limewaweka kwenye mazingira magumu, kwa mfano, kutishiwa na mamlaka za udhibiti. Kwa kuwa baadhi ya maafisa hutaka kupewa pato nyemelezi, matokeo yake ni kuwa baadhi ya mamlaka zimejikuta zikishindwa kutekeleza majukumu yao na kuruhusu wawekezaji kuendelea kufanya shughuli zao kinyume na sheria.

Udhaifu wa uratibu ndani ya serikali pia umeathiri jinsi gani ubia unadhibitiwa linapokuja suala la athari katika mazingira na jamii. Wadau ambao wanaufahamu mkubwa juu ya shughuli za uchimbaji (mfano, Wizara ya Madini na Wataalam katika ngazi ya wilaya) mara nyingi hawashawishiki kuwajulisha mamlaka za udhibiti kama Baraza la Taifa la Hifadhi na Usimamizi wa Mazingira (NEMC) na Wakala wa Usalama na Afya sehemu za kazi (OSHA), ambao hata hivyo hawana rasilimali watu na fedha za kutosha, kuhusu migodi yenye matatizo na yenye kutumia leseni zake kinyume na sheria na kanuni.' Jambo hili linarudisha nyuma kwa kiwango kikubwa juhudi za serikali kusimamia shughuli katika migodi ambazo zinaweza leta athari katika mazingira na usalama kwa wafanyakazi. Hii inaweza kuchukuliwa kama mgongano wa maslahi kati ya maendeleo ya sekta ya madini na ukusanyaji wa mapato kwa upande mmoja na kutimiza malengo ya udhibiti wa masuala ya mazingira na jamii kwa upande mwingine. Hii inatokana na kupishana kwa malengo ambayo hutumika kupima utendaji kazi wa wafanyakazi katika taasisi za udhibiti. Ingawa Mamlaka za Serikali za Mitaa (LGAs) zina wafanyakazi wenye jukumu la kufuatilia ili kuhakikisha usimamizi wa mazingira na masuala ya jamii, wafanyakazi hawa hutakiwa kuripoti kwa

1 Hivi karibuni serikali imetangaza ongezeko la uteuzi wa wakaguzi wa mazingira (kutoka 60 mpaka 435). Wakaguzi hawa ni waajiriwa wa serikali kutoka NEMC, LGAs, na idara nyingine za serikali. Uteuzi unawapa nguvu wakaguzi kusimamisha au kupiga faini shughuli ambayo haifuati sheria au kanuni za mazingira. Bado ni jambo la kusubiri kuona kwamba wakaguzi hawa watapewa rasilimali muhimu zitakazowawezesha kufanya kazi zao za kufuatilia na kuhakikisha utekelezaji wa sheria na kanuni za mazingira. 
mamlaka zao husika ambazo hutoa kipaumbele kwa ajenda za maendeleo ya maeneo yao. Hii hupelekea mawasiliano yenye ufanisi kati ya LGAs na wadau muhimu kama NEMC na OSHA, kwa kiasi kikubwa, kudhoofishwa na siasa za maeneo yao. Zaidi ya hayo, iliyokuwa Wakala wa Ukaguzi wa Madini Tanzania (TMAA) walikuwa na uwezo wa kufanya ukaguzi katika ngazi zote za sekta ya madini, lakini kwa kuwa ufanisi wao hupimwa na uwezo wao wa kukusanya mapato, imepelekea shughuli zao kujikita zaidi kwenye migodi mikubwa ambapo wana uhakika wa kukusanya mrahaba.

Kutokana na gharama na matatizo yanayoweza jitokeza kwa kushindwa kufuata kanuni zilizopo, baadhi ya wawekezaji kwenye sampuli walianza kuwekeza kwenye kurasimisha shughuli zao (mfano, kusajiri maeneo ya kazi, kukata leseni ya uchimbaji mkubwa, na kufuata kanuni za usimamizi wa mazingira) ili kulinda gharama zao za uwekezaji. Kimsingi, hii inabadilisha kabisa jinsi gani wawekezaji wanadhibitiwa, huku taasisi kuu za serikali kama NEMC na OSHA zikichukua majukumu makubwa ya udhibiti. Inadaiwa kuwa utaratibu huu wa kuwekeza nguvu za udhibiti katika ngazi ya serikali kuu ni muhimu kwa uwekezaji wa kigeni kwa sababu taasisi za serikali za ngazi ya chini hazina uwezo wa kudhibiti miradi ya uchimbaji na zinaweza zuiwa na siasa za maeneo yao. Ingawa kuna uzito kwenye hoja hii, kuwekeza nguvu katika serikali kuu kunazinyima nguvu taasisi na mamlaka za ngazi ya chini na kudhoofisha uwezo wa taasisi muhimu za udhibiti kutengeneza mahusiano ya kila siku yanayohitajika kusaidia wawekezaji kuboresha ufanisi wao katika kutekeleza kanuni zinazosimamia usimamizi wa mazingira na jamii.

\section{Mapendekezo}

Tafiti hii imeonesha kwamba ubia kati ya wamiliki wa leseni ya uchimbaji mdogo na wawekezaji wa kigeni umeboresha shughuli za uchimbaji kwenye migodi midogo kwa kutumia teknolojia kubwa hivyo kupelekea migodi kuzalisha kwa wingi kulinganisha na migodi midogo. Ikitumika vizuri, utaratibu wa ubia unaweza kuboresha sekta ya uchimbaji mdogo nchini Tanzania, kushughulikia pengo la ufadhili, na kuinua shughuli za uchimbaji mdogo kwenda uchimbaji wa ngazi ya kati. Hata hivyo, ubia una changamoto za utata wa kisheria, uharibifu wa mazingira, na usalama na afya maeneo ya kazi. lli kuwe na ufanisi katika ubia utakaoleta maendeleo endelevu na kuboresha uchimbaji mdogo, tumeonesha chaguo nne za udhibiti ambazo serikali ya Jamhuri ya Muungano wa Tanzania inaweza kuzingatia. Jedwali 1 limefupisha malengo ya mbinu hizo nne, mahitaji ya udhibiti na ya kitaasisi yanayohusiana na utekelezaji, na fursa na hatarishi kwa ajili ya maendeleo endelevu ya sekta ya Madini nchini. Chaguo hizi hujumuisha kubakisha hali kama ilivyo ya kuruhusu ubia na kuhamasisha wawekezaji kutumia modeli ya biashara inayounganisha badala ya kusambalatisha uchimbaji mdogo; kwa mfano, kupitia makubaliano ya kipekee kwa wawekezaji kuchukua makinikia kwa kubadilishana na msaada wa kitaalamu, mtaji na vitendea kazi.

Jedwali 1. Chaguo za kudhibiti ubia kati ya wachimbaji wadogo na wawekezaji

\begin{tabular}{|c|c|c|c|c|}
\hline & $\begin{array}{l}\text { Option 1: Business } \\
\text { as usual }\end{array}$ & $\begin{array}{l}\text { Option 2: Tougher } \\
\text { enforcement }\end{array}$ & $\begin{array}{l}\text { Option 3: Adapt } \\
\text { existing regulations }\end{array}$ & $\begin{array}{l}\text { Option 3: Alternative } \\
\text { business models }\end{array}$ \\
\hline Objective & $\begin{array}{l}\text { Allow the situation } \\
\text { to evolve organicaly }\end{array}$ & $\begin{array}{l}\text { - Remedy negative } \\
\text { impacts through } \\
\text { formalization }\end{array}$ & $\begin{array}{l}\text { Develop new regulations } \\
\text { to accommodate the } \\
\text { needs of MSM }\end{array}$ & $\begin{array}{l}\text { Promote business models } \\
\text { that integrate rather than } \\
\text { displace ASM activities }\end{array}$ \\
\hline Requirements & $\begin{array}{l}\text { - Avoid disruptive } \\
\text { policies and } \\
\text { regulations }\end{array}$ & $\begin{array}{l}\text { - Investment in gov. } \\
\text { enforcement capacity } \\
\text { - Address internal politics } \\
\text { \& disincetives }\end{array}$ & $\begin{array}{l}\text { - Re-evaluation of } \\
\text { existing regulations } \\
\text { and government } \\
\text { mandates } \\
\text { - Improved dialogue } \\
\text { between government } \\
\text { and MSM }\end{array}$ & $\begin{array}{l}\text { - More involvement of } \\
\text { CSOs in brokering multi- } \\
\text { stakeholder dialogues } \\
\text { - Develop adaptive capacities } \\
\text { and learning amongst } \\
\text { investors, ASM, CSOs, and } \\
\text { governement }\end{array}$ \\
\hline Risks & $\begin{array}{l}\text { - Negative impacts } \\
\text { remain unresolved } \\
\text { - Concentration of } \\
\text { mineral rights by } \\
\text { allowing investors } \\
\text { to accumulate } \\
\text { PMLS }\end{array}$ & $\begin{array}{l}\text { - Investors dtop investing } \\
\text { due to costs } \\
\text { - Investors abandon their } \\
\text { investments } \\
\text { - Widening of infomality } \\
\text { gap between investors } \\
\text { and ASM }\end{array}$ & $\begin{array}{l}\text { - Concentraton of } \\
\text { mineral right by } \\
\text { allowing investors to } \\
\text { accumulate PMLs } \\
\text { - Widening of informality } \\
\text { gap between investors } \\
\text { and ASM }\end{array}$ & $\begin{array}{l}\text { - Investors abandon their } \\
\text { investments } \\
\text { - Power imblanaces if ASM } \\
\text { enters into one-sided } \\
\text { contratct } \\
\text { - Opportunistic trading } \\
\text { practices (e.g. side-selling) } \\
\text { - Loss of value addition }\end{array}$ \\
\hline Opportunities & $\begin{array}{l}\text { - Predictibility } \\
\text { - Investor confidence } \\
\text { - Mineral output } \\
\text { growth }\end{array}$ & $\begin{array}{l}\text { - Improved labor } \\
\text { conditions, } \\
\text { environmental } \\
\text { performance, and public } \\
\text { revenues }\end{array}$ & $\begin{array}{l}\text { - Investor confidence } \\
\text { - Mineral output growth } \\
\text { - Improved social } \\
\text { and environmental } \\
\text { performance }\end{array}$ & \\
\hline
\end{tabular}




\section{Marejeo}

Bryceson DF and Jønsson JB. 2010. Gold digging careers in rural East Africa: Small-scale miners' livelihood choices. World Development 38(3):379-392.

Bryceson DF, Fisher E, Jønsson JB and Mwaipopo R, eds. 2014. Mining and Social Transformation in Africa: Mineralizing and Democratizing Trends in Artisanal Production. London: Routledge.

Carstens J and Hilson G. 2009. Mining and grievance in rural Tanzania. International Development Planning Review 31(3): 301-326.

Chachage CSL. 1995. The meek shall inherit the Earth but not the mining rights: Mining and accumulation in Tanzania. In Gibbon P, ed. Liberalised Development in Tanzania. Uppsala: Reprocentralen HSC.

Fisher E, Mwaipopo R, Mutagwaba W, Nyange D and Yaron G. 2009. The ladder that sends us to wealth: Artisanal mining and poverty reduction in Tanzania. Resources Policy 34(1):1-7.

Hinton J. 2005. Communities and small-scale mining: An integrated review for development planning. Washington, DC: The World Bank.

[HRW] Human Rights Watch. 2013. Toxic toil: Child labor and mercury exposure in Tanzania's small-scale gold mines. Washington DC: HRW.
Jønsson JB and Fold N. 2011. Mining 'from below': Taking Africa's artisanal miners seriously. Geography Compass 5(7):479-93.

Kitula AGN. 2006. The environmental and socio-economic impacts of mining on local livelihoods in Tanzania: A case study of Geita district. Journal of Cleaner Production 14(3-4):405-414.

Kulindwa K, Mashindano O, Shechambo F and Sosovele H. 2003. Mining for Sustainable Development in Tanzania. Dar es Salaam: Dar es Salaam University Press.

Lange S. 2011. Gold and governance: Legal injustices and lost opportunities in Tanzania. African Affairs 110(439):233-252.

[URT] United Republic of Tanzania. 2011. Draft final report on baseline survey on artisanal and small-scale mining (ASM) activities and preparation of an ASM database. Sustainable Management of Mineral Resources Project (SMMRP). Washington DC: World Bank.

\section{Shukrani}

Utafiti huu ulifadhiliwa na Baraza la Utafiti wa Kiuchumi na Kijamii na Idara ya Kimataifa ya Maendeleo kutoka Uingereza, hata hivyo, maoni yaliyotolewa hayawakilishi maoni ya Serikali ya Uingereza. Tathmini hii imefanywa na kampuni ya COWI Tanzania. Shukrani zetu ziwaendee wale wote waliohojiwa kwenye utafiti huu.
The CGIAR Research Program on Forests, Trees and Agroforestry (FTA) is the world's largest research for development program to enhance the role of forests, trees and agroforestry in sustainable development and food security and to address climate change. CIFOR leads FTA in partnership with Bioversity International, CATIE, CIRAD, ICRAF, INBAR and TBI.

\section{iied COWI

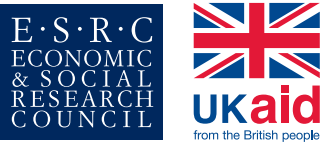

Research jointly supported by the ESRC and DFID 